\title{
A case of diffuse large B-cell lymphoma misdiagnosed as an erysipelas of the face
}

\author{
Ewa Teresiak-Mikołajczak, Magdalena Szymańska, Magdalena Czarnecka-Operacz
}

Department of Dermatology, Poznan University of Medical Sciences, Poland

Head: Prof. Zbigniew Adamski MD, PhD

Postep Derm Alergol 2013; XXX, 4: 268-270

DOI: 10.5114/pdia.2013.37040

\begin{abstract}
We report a case of a woman with diffuse large B-cell lymphoma (DLBCL). Primary cutaneous lymphomas (PCLs) represent distinct clinical and histopathologic subtypes of extranodal T- and B-cell lymphomas. Cutaneous B-cell lymphomas comprise $20-25 \%$ of all primary cutaneous lymphomas. The patient presented an erythematous tumour mass of the left nasolabial fold, nose and left cheek as well as disseminated infiltrative plagues on the trunk, arms and left lower leg. Skin biopsy revealed a diffuse infiltrate of lymphocytes around hair follicles and blood vessels within dermis and subcutaneous tissue. An immunohistochemistry showed a diffuse infiltrate of large non-cleaved B-cells, with a high proportion of centroblast-like cells within dermis. Tumor cells expressed CD20, bcl-2 protein and did not express CD10. The patient was misdiagnosed as the erysipelas of the face and unsuccessfully treated with long-term antibiotic therapy by a laryngologist and a dermatologist. The correct diagnosis was delayed and established after 6 months' history of DLBCL lesions. Therefore, we would like to strongly stress the importance of considering diagnosis of cutaneous lymphomas in chronic skin lesions non-responsive to adequate therapies.
\end{abstract}

Key words: diffuse large B-cell lymphoma, primary cutaneous lymphomas, cutaneous B-cell lymphoma.

\section{Introduction}

Primary cutaneous lymphomas (PCLs) represent distinct clinical and histopathologic subtypes of extranodal T- and B-cell lymphomas. Until recently, a distinction between primary and secondary cutaneous lymphomas has not been characterized. Therefore, both groups of lymphomas were classified according to histologic classification schemes used by hematologists for nodal non-Hodgkin lymphomas such as Kiel or the Revised European-American Lymphoma (REAL) classification [1, 2]. Recently, the European Organization for Research and Treatment of Cancer (EORTC) has proposed a new classification of PCLs based on a combination of clinical, histologic, and immunophenotypic criteria. The new classification allows for a more uniform diagnosis and treatment of PCLs [3]. Primary cutaneous lymphomas are defined as patients without concurrent extracutaneous disease at the time of diagnosis. Primary cutaneous lymphomas often present highly characteristic clinical and histologic features, clinical course and prognosis, which are different from primary nodal lymphomas of the same histologic subtype, involving the skin secondarily [3].
Cutaneous B-cell lymphomas comprise $20-25 \%$ of all primary cutaneous lymphomas [4-6]. Primary cutaneous diffuse large B-cell lymphomas (PCDLBCL) are composed of large $B$ cells (centroblasts and immunoblasts). Two forms of PCDLBCL are distinguished in the WHO-EORTC classification: DLBCL, leg-type and DLBCL, other. The most common variant, DLBCL, leg-type, usually occurs on the leg, however it may affect other sites as well. Other variants referred to as DLBCL-other comprise T-cell/histiocyterich $\mathrm{DLBCL}$, plasmablastic lymphoma and others that do not fulfill the criteria for DLBCL, leg-type [5, 7].

\section{Case report}

A 65-year-old woman was referred to the Dermatology Department for diagnosis and treatment of disseminated, infiltrative plaques of the left cheek and trunk. First symptoms presented as impaired nasal drainage and erythematous plague of the left nasolabial fold and cheek appeared in December 2011. In March 2012, disseminated red plagues appeared also on the upper trunk and arms, as well as one solitary lesion on the left calf. The patient

Address for correspondence: Ewa Teresiak-Mikołajczak MD, PhD, Department of Dermatology, Poznan University of Medical Sciences, 49 Przybyszewskiego St, 60-355 Poznan, Poland, phone: +48 601957 851, e-mail: ewa.teresiak@wp.pl Received: 30.11.2012, accepted: 10.02.2013. 
was first diagnosed by a family doctor as sinusitis and treated with systemic clindamycin. In January, the patient was consulted by a laryngologist who diagnosed erysipelas although no typical systemic symptoms were present. The patient was then referred to a dermatologist who continued systemic antibiotic therapy with doxycycline and amoxicillin with no clinical improvement.

At the moment of admission to the Dermatology Department, we observed an infiltrative, erythematous tumour mass situated on the left nasolabial fold, nose and left cheek (Figures 1 and 2) as well as disseminated infiltrative plagues on the trunk, arms (Figure 3) and left calf. The patient complained of impaired nasal drainage and burning sensation within skin lesions. Magnetic resonance imaging of the facial skeleton and nasal sinuses revealed neoplastic mass/tumour of the soft tissues of the left nose and cheek invading bones of the nose, left nasal cavity and left ethmoid sinus. Skin biopsy from both cheek and the leg showed a diffuse infiltrate of lymphocytes around hair follicles and blood vessels within dermis and subcutaneous tissue. An immunohistochemistry revealed a diffuse infiltrate of large non-cleaved B-cells, with a high proportion of centroblast-like cells within dermis. Tumor cells expressed CD20, bcl-2 protein and did not express CD10. The staging of the disease was performed and no signs of extracutaneous lymphomas were found. So the diagnosis of diffuse large B-cell lymphoma was finally clarified. The patient was referred to the Department of Hematology for further treatment with chemotherapy.

\section{Discussion}

Most patients affected by PCDLBCL are elderly with a female predominance. The peak of incidence is in the seventh decade of life [4]. According to the WHO classification, DLBCL are a heterogeneous disorder including several disease entities. Although the WHO classification does not identify PCDLBCL as a specific subtype it recommends that the site of presentation may be a marker for underlying biologic differences between morphologically similar entities. The EORTC classifies PCLs mainly on the basis of anatomic location. Diffuse large B-cell lymphomas is most common on the legs and is characterized by a relatively aggressive course and poor prognosis. In turn, primary cutaneous follicular lymphomas (PCFL) occur on the upper body, particularly the head and neck, and have a good prognosis [8].

Clinically DLBCL is characterized by rapidly growing red or violet tumour masses localized mostly on lower extremities, but they may affect sites other than legs [5]. The diagnosis is based on the histopathological examination. Histopathologically, characteristic findings include diffuse non-epidermotropic infiltrates of cutaneous and subcutaneous tissue. The infiltrates are predominantly composed of medium/large monomorphic non-cleaved B cells, with variable proportions of centroblast- and immunoblast-like

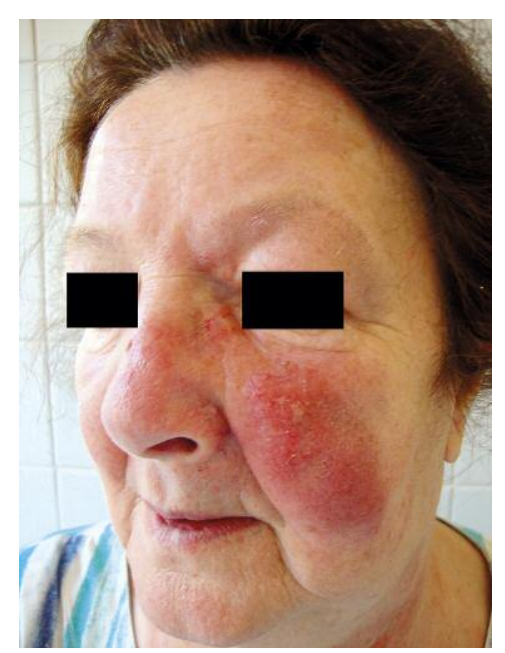

Fig. 1. An infiltrative, erythematous tumour mass situated on the left nasolabial fold, nose and left cheek

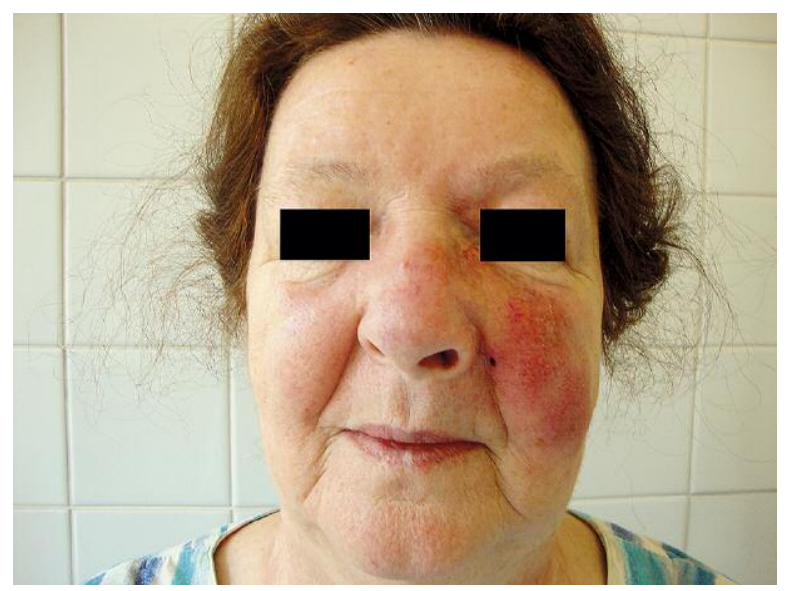

Fig. 2. An infiltrative, erythematous tumour mass situated on the left nasolabial fold, nose and left cheek causing deformation of the face (deviation of the nose to the right side)

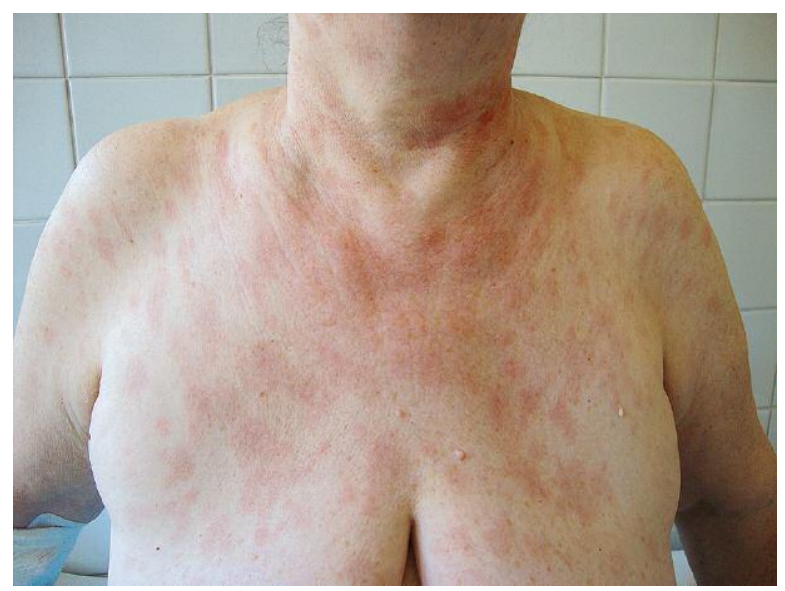

Fig. 3. Disseminated infiltrative plagues on the upper trunk and arms 
cells with non-cleaved nuclei [5, 9]. Neoplastic cells express CD20, bcl-2 and CD79a. DLBCL in most cases express bcl-6, but do not express CD10, which helps to differentiate DLBCL from PCFCL (primary cutaneous follicular lymphoma) [5].

Patients with DLBCL, especially leg type, differ from patients with other types of primary cutaneous B-cell lymphomas by higher age of onset, a poorer prognosis and constant expression of bcl-2 protein [10]. A general 5-year survival rate is $20-55 \%$ [4]. The number of skin lesions at diagnosis is the most important prognostic factor in these patients. The 5 -year survival rate is 100\% in patients suffering from DLBCL with solitary skin lesions. Five-year life expectancy regards $45 \%$ patients with numerous skin lesions confined to one area, but only $36 \%$ of patients with DLBCL affecting two distinct areas (e.g. both extremities). A poor prognostic sign is also loss of p16 expression $[4,10]$.

Patients with DLBCL should be examined thoroughly for nodal and extracutaneous disease, because possible involvement of internal organs is significant [4]. Treatment options include radiotherapy and chemotherapy. In patients with a solitary skin tumour, radiotherapy may be considered as a first choice treatment. In patients with multifocal skin lesions or relapses, chemotherapy is recommended. Cyclophosphamide, doxorubicin, vincristine and prednisone (CHOP) is the standard chemotherapy for the disease with a cure rate of $40 \%$ to $50 \%$. New therapeutic options include a combination of CHOP and rituximab. Rituximab is an antiCD20 monoclonal antibody and eliminates neoplastic B cells by induction of apoptosis, activation of complement and antibody-dependent cellular cytotoxicity $[5,9,11]$.

Diffuse large B-cell lymphomas is a very rare disorder, however associated with a poor prognosis. Cutaneous lymphomas may clinically manifest as different skin disorders e.g. chronic venous ulceration $[9,12,13]$. Our patient was misdiagnosed as the erysipelas of the face and unsuccessfully treated with long-term systemic therapy with antibiotics by a laryngologist and also a dermatologist. The correct diagnosis was delayed and established after 6 months of persisting DLBCL lesions. Therefore, we want to underline the importance of considering a possible diagnosis of cutaneous lymphomas in chronic skin lesions nonresponsive to adequate therapies. Unfortunately, patients with cutaneous lymphomas in Poland may be treated by hematologists only, so our patient has been immediately directed to the Department of Hematology of the Medical University in Poznan.

\section{References}

1. Stansfeld AG, Diebold J, Kapanci Y, et al. Updated Kiel classification for lymphomas. Lancet 1988; 1: 292-3.

2. Harris NL, Jaffe ES, Stein H. A revised European-American classification of lymphoid neoplasms: a proposal from the International Lymphoma Study Group. Blood 1994; 84: 1361-92

3. Willemze R, Kerl H, Sterry W, et al. EORTC classification for primary cutaneous lymphomas: a proposal from the cutaneous lymphoma study group of the European Organization for Research and Treatment of Cancer. Blood 1997; 90: 354-71.

4. Burgdorf WHC, Plewig G, Wolff HH, et al. Braun-Falco's Dermatology. Springer MedizinVerlag, Heidelberg 2009; 1493.

5. Sokołowska-Wojdyło M, Roszkiewicz J. Primary cutaneous lymphomas. Czelej, Lublin 2008; 37.

6. Sokołowska-Wojdyło M, Sikorska M, Florek A, et al. Lymphomas of the head and neck in dermatological practice. Postep Derm Alergol 2012; 29: 313-20.

7. Burg G, Kempf W, Cozzio A, et al. WHO-EORTC classification of cutaneous lymphomas 2005: histological and molecular aspects. J Cutan Pathol 2005; 32: 647-74.

8. Goodlad JR, Krajewski AS, Batstone PJ, et al. Primary cutaneous diffuse large B-cell lymphoma. Prognostic signifficance of clinicopathological subtypes. Am J Surg Pathol 2003; 27: 1538-45.

9. Carlesimo M, Orsini AN, Abruzzese C, et al. A case of cutaneous large B-cell lymphoma of the legs appearing as chronic venous ulceration. Hematol Rep 2012; 4: 17-8.

10. Grange F, Bekkenk MW, Wechsler J, et al. Prognostic factors in primary cutaneous large B-cell lymphomas: a European multicenter study. J Clin Oncol 2001; 19: 3602-10.

11. Wojewoda K, Brenner J, Sokołowska-Wojdyło M, BarańskaRybak W. Treatment of primary cutaneous lymphoma with reference to the latest therapeutic consensus of the Polish Lymphoma Research Group (PLRG). Postep Derm Alergol 2012; 29: 63-8.

12. Kreft B, Bednarczyk M, Emmerling F, Marsch WC. Cutaneoussubcutaneous pseudolymphoma after specific immunotherapy with grass-rye pollen-allergen extract containing aluminium hydroxide. Postep Derm Alergol 2011; 28: 134-7.

13. Sokołowska-Wojdyło M, Malek M, Majewska H, et al. Ichthyosis as a paraneoplastic syndrome and atypical variant of mycosis fungoides: two cases report. Postep Derm Alergol 2012; 29: 467-70. 